\title{
Right ventricular end-systolic remodeling index on cardiac magnetic resonance imaging: comparison with other functional markers in patients with chronic thromboembolic pulmonary hypertension
}

\author{
Ling Zhang ${ }^{1}$, Jinzhu Dai ${ }^{1}$, Peiyao Zhang ${ }^{1}$, Haiyi Ma ${ }^{1}$, Xincao Tao ${ }^{2}$, Yanan Zhen ${ }^{3}$, Xiaopeng Liu ${ }^{3}$, \\ Wanmu Xie ${ }^{2}$, Jun Wan ${ }^{2}$, Min Liu ${ }^{1}$ \\ ${ }^{1}$ Department of Radiology, China-Japan Friendship Hospital, Beijing, China; ${ }^{2}$ Department of Pulmonary and Critical Care Medicine, China-Japan \\ Friendship Hospital, Beijing, China; ${ }^{3}$ Department of Cardiovascular Surgery, China-Japan Friendship Hospital, Beijing, China
}

Contributions: (I) Conception and design: M Liu, J Wan; (II) Administrative support: M Liu; (III) Provision of study materials or patients: X Tao, Y Zhen, X Liu, W Xie, J Wan; (IV) Collection and assembly of data: L Zhang, J Dai, P Zhang, H Ma; (V) Data analysis and interpretation: L Zhang, P Zhang, M Liu; (VI) Manuscript writing: All authors; (VII) Final approval of manuscript: All authors.

Correspondence to: Min Liu. 2 Yinghua Dong Street, Hepingli, Chao Yang District, Beijing 100029, China. Email: drradiology@163.com.

Background: Cardiac magnetic resonance imaging (CMR) can provide important metrics of pulmonary hypertension. In the current study, we investigated whether the CMR-derived right ventricular end-systolic remodeling index (RVESRI) could be a metric in assessing the function and hemodynamics of chronic thromboembolic pulmonary hypertension (CTEPH).

Methods: A total of 64 patients ( $45 \pm 14$ years, 37 males), including 46 patients with CTEPH and 18 patients with chronic pulmonary thromboembolism (CTE), were retrospectively enrolled. All patients underwent right heart catheterization and CMR within 7 days. RVESRI, right ventricular eccentricity index, right ventricular end-diastolic and end-systolic volume index, right ventricular ejection fraction, right ventricular cardiac output, and strain were analyzed on cine images of CMR. Hemodynamic parameters including mean pulmonary arterial pressure, pulmonary vascular resistance, and cardiac output were obtained from right heart catheterization.

Results: RVESRI of all patients was 1.50 (IQR, 1.26-1.90). Compared with CTE patients, RVESRI in patients with CTEPH was significantly increased $(\mathrm{U}=27.5, \mathrm{P}<0.001)$. The interclass correlation coefficients of intra-observer reproducibility and inter-observer reproducibility for RVESRI measurement were 0.96 (95\% CI, 0.93-0.97) and 0.99 (95\% CI, 0.98-0.99), respectively. RVESRI positively correlated with right ventricular end-diastolic and end-systolic volume index and right ventricular global longitudinal strain $(\mathrm{r}=0.79,0.83,0.62, \mathrm{P}<0.001)$, while it was negatively correlated with right ventricular ejection fraction $(r=-0.64, \mathrm{P}<0.001)$, right ventricular cardiac output $(\mathrm{r}=-0.50, \mathrm{P}<0.001)$, and right ventricular eccentricity index $(r=-0.81, P<0.001)$. RVESRI had a positive correlation with mean pulmonary arterial pressure $(r=0.65$, $\mathrm{P}<0.001)$ and pulmonary vascular resistance $(\mathrm{r}=0.69, \mathrm{P}<0.001)$, while it was negatively correlated with cardiac output $(\mathrm{r}=-0.64, \mathrm{P}<0.001)$. The receiver operating characteristic curve indicated that RVESRI $>1.35$ had a sensitivity of $97.8 \%$ and specificity of $83.3 \%$ in predicting mean pulmonary arterial pressure $\geq 25 \mathrm{mmHg}$, and its area under the curve (AUC) was $0.96 \pm 0.02$. Meanwhile, the AUC of RVESRI was similar to RVEI $(\mathrm{Z}=1.635, \mathrm{P}=0.102)$ and was more than the diameter of the main pulmonary artery $(\mathrm{MPA})(\mathrm{Z}=2.26, \mathrm{P}=0.02)$ and the ratio of the MPA and ascending aorta diameter (MPA/AAo) $(\mathrm{Z}=3.826, \mathrm{P}<0.001)$ in predicting mean pulmonary arterial pressure $\geq 25 \mathrm{mmHg}$.

Conclusions: RVESRI measured on CMR is a simple and reproducible metric in assessing right ventricular function and hemodynamics in CTEPH patients. 
Keywords: Chronic thromboembolic pulmonary hypertension; cardiovascular magnetic resonance; RV endsystolic remodeling index; right ventricular function; hemodynamics

Submitted Apr 08, 2021. Accepted for publication Aug 04, 2021.

doi: 10.21037 /qims-21-385

View this article at: https://dx.doi.org/10.21037/qims-21-385

\section{Introduction}

Chronic pulmonary thromboembolism (CTE) (1-3) results from incomplete lysis of acute pulmonary embolism with the subsequent organizationof residual thrombus. It may lead to pulmonary arterial pressure, pulmonary vascular resistance, and right heart dysfunction, which is called chronic thromboembolic pulmonary hypertension (CTEPH). The right ventricle and pulmonary vasculature are integrally related, and measures of right ventricular afterload, stress, and functional adaptation provide important prognostic information for patients with pulmonary hypertension (PH) (4). This is also extremely important for patients with CTEPH.

Echocardiography is the tool most widely available for monitoring the ventricular function of patients with $\mathrm{PH}$. Amsallem et al. (5) reported that the right ventricular endsystolic remodeling index (RVESRI) measured on the right ventricular apical 4-chamber echocardiographic view strongly predicted outcomes in pulmonary arterial hypertension (PAH). However, the technique has several limitations that limit the accurate evaluation of right ventricular performance, especially operator experience dependence and patientdependent echo windows. Due to the high spatial resolution and the independence of the doctor's experience, cardiac magnetic resonance imaging (CMR) can show the anatomical complexity of the right ventricle and quantify ventricular function, blood flow, myocardial perfusion, and fibrosis (6-8). So far, the application of RVESRI measured on CMR in the assessment of CTEPH has not been investigated. Thus, the current objective was to assess the reproducibility of RVESRI measured with CMR to determine whether RVESRI correlates with other CMR functional parameters and hemodynamics measured with right heart catheterization (RHC) in patients with CTEPH.

\section{Methods}

\section{Study design}

This study was registered at the Chinese Clinical Trials
Registry Center (http://www.chictr.org/en/; registration number ChiCTR-OCH-14004929) and was approved by our institutional review board (IRB No. 2017-24). The study was conducted following the Declaration of Helsinki (as revised in 2013), and all patients signed informed consents. We retrospectively included 64 patients $(45 \pm 14$ years, 37 males), including 46 patients with CTEPH and 18 with CTE from January 2017 to January 2020. All patients who underwent RHC and CMR within 1 week in our hospital were included. CTEPH was diagnosed by tissue pathology from pulmonary endarterectomy, characteristic findings on CT pulmonary angiography or ventilation-perfusion scintigraphy, and elevated pulmonary arterial pressure and pulmonary vascular resistance measured with RHC. CTE was diagnosed by a prior history of acute pulmonary embolism, characteristic findings on CT pulmonary angiography or ventilation-perfusion scintigraphy, and normal pulmonary arterial pressure and pulmonary vascular resistance measured with RHC. The flowchart of this study is shown in Figure 1.

\section{CMR}

All patients underwent CMR on a 1.5 Tesla clinical MR scanner (MAGNETOM Area, Siemens Healthcare, Erlangen, Germany) with an 18-channel phased-array surface coil. All images were acquired with retrospective electrocardiographic gating in the end-expiratory breath hold. Standard contiguous short-axis slices covering both ventricles from base to apex and long-axis 4-chamber cine images were acquired with the balanced steadystate free precession sequence. The typical acquisition parameters included repetition time/echo time $34.5 / 1.1 \mathrm{~ms}$, flip angle $50-60^{\circ}$, slice thickness $6 \mathrm{~mm}$, in-plane spatial resolution $1.8 \times 1.8 \mathrm{~mm}^{2}$, temporal resolution $40 \mathrm{~ms}$, and 25 reconstructed cardiac phases.

\section{CMR analysis}

RVESRI is the ratio of the lateral free wall length to 


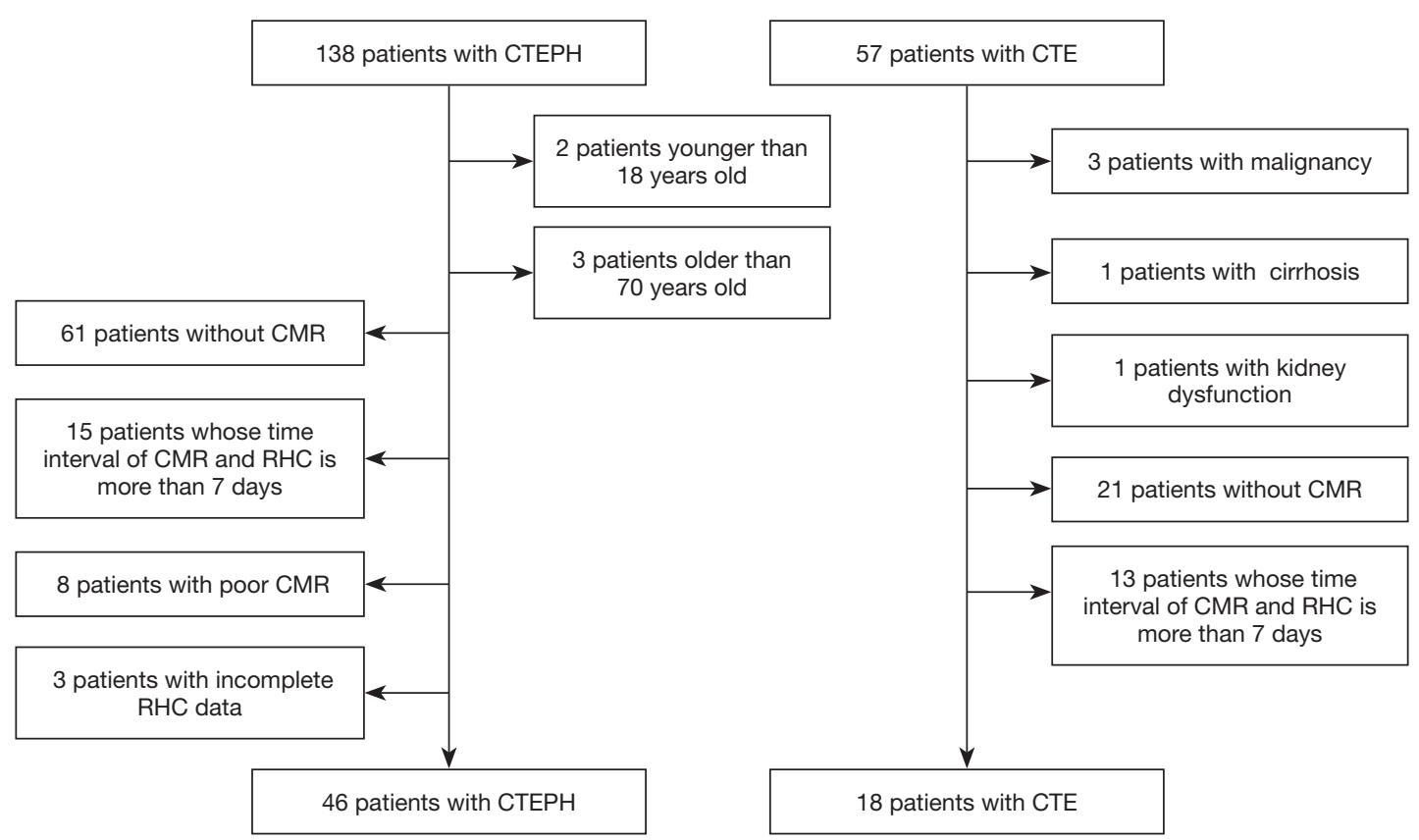

Figure 1 The flowchart of the study. CTEPH, chronic thromboembolic pulmonary hypertension; CTE, chronic pulmonary thromboembolism; CMR, cardiac magnetic resonance imaging; RHC, right heart catheterization.

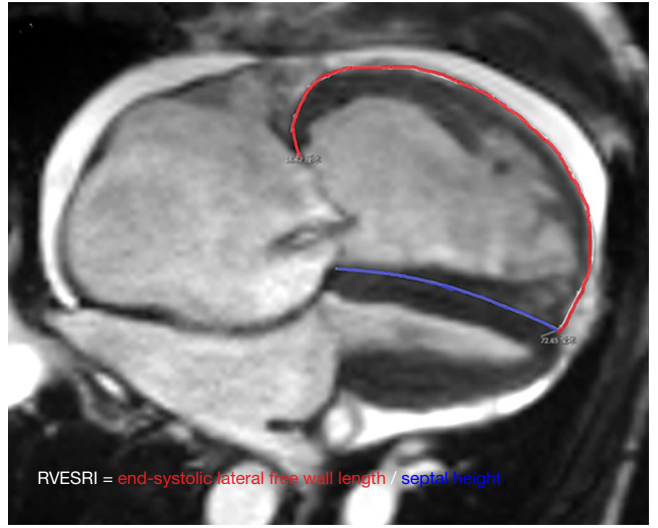

Figure 2 Measurement of right ventricular end-systolic remodeling index (RVESRI) on the 4-chamber cine image of cardiac magnetic resonance imaging (CMR) in end systole. RVESRI was the ratio of end-systolic lateral free wall length (red line) to interventricular septal height (blue line).

septal wall height in the end-systolic phase (5) and was independently measured on the long-axis 4-chamber cine image of CMR (Figure 2) by 2 residents with 5 years of experience on the picture archiving and communication system. After one month, the measurement of RVESRI was repeated by one resident. Moreover, the right ventricular eccentricity index (RVEI) (9) was also calculated on shortaxis cine images at the mid ventricular level of the right ventricle in the end systole by one resident with 4 years of experience.

RV functional metrics were measured on the short-axis and long-axial 4-chamber cine images with the commercial software, CardiacFunction, on SyngoVia workstation (Siemens Healthcare, Erlangen, Germany) by a resident with 4 years ofexperience. Right and left ventricular epicardial and endocardial surfaces were traced from the contiguous short-axis cine images. End-diastole and endsystole, respectively were defined as the first cine phase of the R-wave triggered acquisition or the largest volume and the smallest cavity area. From the end-diastolic and end-systolic volumes, right ventricular and left ventricular end-diastolic and end-systolic volumes/body surface area (RVEDVI, RVESVI, LVEDVI, LVESVI), right ventricular and left ventricular ejection fractions (RVEF and LVEF, respectively), cardiac output (CO), and right ventricular myocardial mass index (RVMI) were calculated.

Moreover, RV strain including right ventricular global longitudinal strain (RVGLS), circumferential strain (RVGCS), and radial strain (RVGRS) was analyzed from cine images in the 4-chamber view and short-axial views using $\mathrm{CVI}^{42}$ (Version 5.11, Circle Cardiovascular Imaging 
Inc., Calgary, Alberta, Canada) by a resident with 4 years of experience.

\section{Hemodynamics}

Hemodynamics were evaluated with RHC. Venous access was obtained by inserting an introducer into the internal jugular vein. A Swan-Ganz standard thermodilution pulmonary artery catheter was placed at the right inferior pulmonary artery. The metrics included diastolic pulmonary arterial pressure (DPAP), systolic pulmonary arterial pressure (SPAP), mean pulmonary arterial pressure (MPAP), right atrial pressure (RAP), pulmonary capillary wedge pressure (PCWP), and pulmonary vascular resistance (PVR). $\mathrm{CO}$ was determined using the Fick method.

\section{Statistical analysis}

Statistical analysis was performed using SPSS 22 (Chicago, IL, USA) and MedCalc statistical software (version15.6.1, Ostend, Belgium). Data are expressed as mean \pm standard deviation (SD) or median with interquartile range (IQR). Bland-Altman plots and the intraclass correlation coefficient (ICC) for RVESRI were calculated for the intra- and interobserver agreement. The differences in RV functional parameters, including RVESRI between CTEPH and CTE, were compared with the nonparametric Mann-Whitney U test. Spearman's rank test was used to evaluate correlations between RVESRI and other RV functional parameters, plasma biomarkers, and hemodynamic metrics. A receiver operating characteristic (ROC) curve was used to analyzethe area under the curve (AUC) of the ROC curve and measure cut-off values of RVESRI, RVEI, the diameter of the main pulmonary artery (MPA), and the ratio of the MPA and ascending aorta diameter (MPA/AAo) in predicting MPAP $\geq 25 \mathrm{mmHg}$. Statistical comparisons of ROC curves were analyzed with the DeLong test (MedCalc statistical software). A P value $<0.05$ was considered statistically significant.

\section{Results}

\section{Baseline characteristics}

The demographic, clinical, and hemodynamic-derived information of all patients are shown in Table 1. There were no differences among CTEPH and CTE patients concerning age, sex, body mass index (BMI), systolic blood pressure (SBP), and diastolic blood pressure (DBP). A total of 19 patients $(29.7 \%)$ were classified as World Health Organization (WHO) functional class I, $26(40.6 \%)$ patients as WHO class II, patients $16(25.0 \%)$ as WHO class III, and $3(4.6 \%)$ patients as WHO class IV. Table 2 showed there was a significant difference between the RVESRI and other CMR functional metrics in patients with CTEPH and CTE, except for RVGRS, RVGCS, LVESVI, and LVEF.

\section{Reproducibility of RVESRI measurements}

A Bland-Altman plot (Figure $3 A$ ) of the RVESRI measurements from the 2 observers showed that the bias (difference between the means) was 0 and the $95 \%$ limits of agreement were between -0.12 and 0.11. A BlandAltman plot (Figure 3B) of RVESRI measurements repeated by one observer showed that the bias (difference between the means) was 0 and the $95 \%$ limits of agreement were between -0.13 and 0.13 . The ICC for intra-observer reproducibility was 0.96 [95\% confidence interval (CI), 0.93-0.97] for RVESRI. The ICC for inter-observer reproducibility was 0.99 (95\% CI, 0.98-0.99) for RVESRI.

\section{Correlation of RVESRI with RV functional metrics and plasma biomarkers}

As shown in Table 3, RVESRI positively correlated with RVEDVI, RVESVI, RVMI, and RVGLS, while RVESRI negatively correlated with RVEI, RVEF, and RVCO in the total patients and CTEPH patients. RVESRI did not correlate with RVGRS or RVCRS. Figure 4 shows that RVESRI positively correlated with plasma $\mathrm{N}$-terminal probrain natriuretic peptide (NT-proBNP) $(\mathrm{r}=0.85, \mathrm{P}<0.001)$ and negatively correlated with six-minute walk distance (6MWD) $(\mathrm{r}=-0.79, \mathrm{P}<0.001)$. RVESRI also correlated with cardiac troponin $\mathrm{T}(\mathrm{r}=0.53, \mathrm{P}<0.001)$ but did not correlate with $\mathrm{D}$-dimer $(\mathrm{r}=0.06, \mathrm{P}=0.65)$ or creatine kinase isoenzyme $(r=0.16, P=0.23)$. Furthermore, in patients with CTEPH, RVESRI positively correlated with plasma NT-proBNP $(\mathrm{r}=0.75, \mathrm{P}<0.001)$ and negatively correlated with $6 \mathrm{MWD}$ $(\mathrm{r}=-0.50, \mathrm{P}=0.001)$.

\section{Correlations of RVESRI with bemodynamics}

Figure 5 shows that RVESRI positively correlated with RAP $(r=0.64, \mathrm{P}<0.001)$, MPAP $(\mathrm{r}=0.65, \mathrm{P}<0.001)$, and PVR $(r=0.69, P<0.001)$, while there was a negative correlation between RVESRI and CO $(r=-0.64, \mathrm{P}<0.001)$ in all patients. 
Table 1 Clinical data of all included patients

\begin{tabular}{|c|c|c|c|c|}
\hline Characteristic & Total & CTEPH & CTE & $\chi^{2} / U(P)$ \\
\hline Male/female & $37 / 27$ & $27 / 19$ & $10 / 8$ & $\chi^{2}=0.052(0.518)$ \\
\hline Age (years), mean $\pm S D$ & $45 \pm 14$ & $46 \pm 13$ & $41 \pm 16$ & $329.5(0.206)$ \\
\hline Heart rate $(b / m i n)$, mean $\pm S D$ & $84 \pm 16$ & $84 \pm 17$ & $84 \pm 12$ & $398.5(0.816)$ \\
\hline Systolic BP $(\mathrm{mmHg})$, mean $\pm \mathrm{SD}$ & $117 \pm 14$ & $116 \pm 15$ & $120 \pm 5$ & $322.5(0.212)$ \\
\hline Diastolic BP $(\mathrm{mmHg})$, mean $\pm \mathrm{SD}$ & $79 \pm 11$ & $77 \pm 11$ & $80 \pm 9$ & $355.0(0.376)$ \\
\hline Six-minute walk distance (m), median (IQR) & $360(200-500)$ & $305(200-381)$ & $620(553-683)$ & $9.50(<0.001)^{\star}$ \\
\hline \multicolumn{5}{|l|}{ Plasma biomarkers } \\
\hline CTNT (ng/mL), median (IQR) & $0.007(0.004-0.014)$ & $0.011(0.006-0.030)$ & $0.001(0.001-0.006)$ & $129.0(<0.001)^{\star}$ \\
\hline CKMB (ng/mL), median (IQR) & $1.23(0.72-2.25)$ & $1.69(1.00-2.32)$ & $0.55(0.37-1.04)$ & $182.5(0.001)$ \\
\hline \multicolumn{5}{|l|}{ Hemodynamics } \\
\hline RAP (mmHg), median (IQR) & $3.0(-1-10.5)$ & $4.0(1.0-12.0)$ & $-2.0(-2.0-0.5)$ & $56.5(<0.001)^{\star}$ \\
\hline Systolic PAP (mmHg), median (IQR) & $73.0(27.0-85.0)$ & $80.0(67.0-87.0)$ & $23.5(19.0-27.0)$ & $27.5(<0.001)^{\star}$ \\
\hline Diastolic PAP (mmHg), median (IQR) & $24.0(6.0-39.0)$ & $29.5(23.0-39.0)$ & $5.0(5.0-6.0)$ & $31.0(<0.001)^{\star}$ \\
\hline Mean PAP (mmHg), median (IQR) & $40.0(14.0-54.8)$ & $44.5(39.0-54.3)$ & $12.5(10.0-14.0)$ & $26.0(<0.001)^{\star}$ \\
\hline PCWP (mmHg), median (IQR) & $7.5(4.3-9.0)$ & $8.0(7.0-10.3)$ & $5.0(1.0-6.5)$ & $164.5(0.004)$ \\
\hline Combination medical therapy & 10 & 2 & 8 & \\
\hline
\end{tabular}

*, $\mathrm{P}<0.001$, comparison of CMR metrics between CTEPH patients and CTE patients. BP, blood pressure; CTEPH, chronic thromboembolic pulmonary hypertension; CTE, chronic pulmonary thromboembolism; NT-proBNP, N-terminal pro-brain natriuretic peptide; CTNT, cardiac troponin $\mathrm{T}$; CKMB, creatine kinase isoenzyme; RAP, right atrial pressure; PAP, pulmonary arterial pressure; PCWP, pulmonary capillary wedge pressure; PVR, pulmonary vascular resistance; $\mathrm{CO}$, cardiac output.

RVESRI did not correlate with PCWP ( $\mathrm{r}=0.20, \mathrm{P}=0.139)$. In patients with CTEPH, RVESRI positively correlated with MPAP ( $\mathrm{r}=0.33, \mathrm{P}=0.01)$ and PVR $(\mathrm{r}=0.42, \mathrm{P}=0.003)$, while RVESRI was negatively correlated with $\mathrm{CO}(\mathrm{r}=-0.52$, $\mathrm{P}<0.001)$. RVESRI in patients with CTEPH did not correlate with RAP $(r=0.24, \mathrm{P}=0.053)$ or PCWP $(r=0.16$, $\mathrm{P}=0.211$ ). Furthermore, Figure 6 shows the ROCs of
RVESRI, RVEI, RVMI, MPA, and MPA/AAo in predicting MPAP $\geq 25 \mathrm{mmHg}$. Table 4 indicates that the AUC of RVESRI was comparable to RVEI and was more than RVMI, MPA, and MPA/AAo ratio. RVESRI $>1.35$ (optimal ROC cutoff) had a sensitivity of $97.8 \%$ and a specificity of $83.3 \%$ for predicting MPAP $\geq 25 \mathrm{mmHg}$, with an AUC of $0.96 \pm 0.02$ (95\% CI, 0.926-1.000). 
Table 2 The comparison of CMR metrics between patients with CTEPH and CTE

\begin{tabular}{|c|c|c|c|c|}
\hline CMR metrics & Total $(n=64)$ & CTEPH $(n=46)$ & CTE $(n=18)$ & $U(P)$ \\
\hline RVEDVI (mL/m²) & 85.7 (69.6-121.3) & $96.4(81.5-125.6)$ & $69.9(55.0-73.9)$ & $121.0(P<0.001)^{\star}$ \\
\hline RVESVI (mL/m²) & $47.5(37.8-80.1)$ & $56.8(46.7-84.1)$ & $29.0(20.2-37.7)$ & $55.1(\mathrm{P}<0.001)^{\star}$ \\
\hline RVEF (\%) & $45.8(26.7-56.7)$ & $36.8(17.0-48.0)$ & $55.4(46.8-64.9)$ & $137.0(P<0.001)^{\star}$ \\
\hline $\operatorname{RVMI}\left(g / m^{2}\right)$ & $23.2(12.8-27.3)$ & $24.0(15.5-29.9)$ & $10.4(9.1-13.3)$ & $98.5(\mathrm{P}<0.001)^{\star}$ \\
\hline RVEI & $1.9(1.6-2,6)$ & $1.7(1.6-2.0)$ & $2.8(2.7-3.0)$ & $12.5(P<0.001)^{*}$ \\
\hline RVGLS (\%) & $-16.1(-20.6--8.8)$ & $-11.9(-16.9--6.2)$ & $-22.2(-23.2--18.7)$ & $78.5(\mathrm{P}<0.001)^{\star}$ \\
\hline RVGRS (\%) & $12.1(8.9-14.1)$ & $11.3(7.5-14.1)$ & $12.3(11.5-16.0)$ & $311.5(P=0.126)$ \\
\hline LVESVI $\left(\mathrm{mL} / \mathrm{m}^{2}\right)$ & $27.5(21.0-30.6)$ & $27.5(20.6-31.7)$ & $27.1(25.9-29.7)$ & $379.0(P=0.692)$ \\
\hline LVEF (\%) & $57.2(46.1-62.2)$ & $56.3(43.8-61.5)$ & $61.6(56.3-62.6)$ & $288.0(P=0.062)$ \\
\hline MPA (mm) & 33.3 (28.3-35.9) & $35.0(32.0-37.5)$ & $28.3(25.8-31.0)$ & $126.5(\mathrm{P}<0.001)^{\star}$ \\
\hline
\end{tabular}

*, $\mathrm{P}<0.001$, comparison of CMR metrics between CTEPH patients and CTE patients. Values are expressed as median and interquartile range (IQR). CTEPH, chronic thromboembolic pulmonary hypertension; CTE, chronic pulmonary thromboembolism; RVESRI, RV end-systolic remodeling index; RVEDVI, right ventricular end-diastolic volume index; RVESVI, right ventricular end-systolic volume index; RVEF, right ventricular ejection fraction; RVCO, right ventricular cardiac output; RVMI, right ventricular myocardial mass index; RVEI, right ventricular eccentricity index; RVGLS, right ventricular global longitudinal strain; RVGRS, right ventricular global radial strain; RVGCS, right ventricular global circumferential strain; LVEDVI, left ventricular end-diastolic volume index; LVESVI, left ventricular end-systolic volume index; LVEF, left ventricular ejection fraction; MPA, main pulmonary artery diameter.
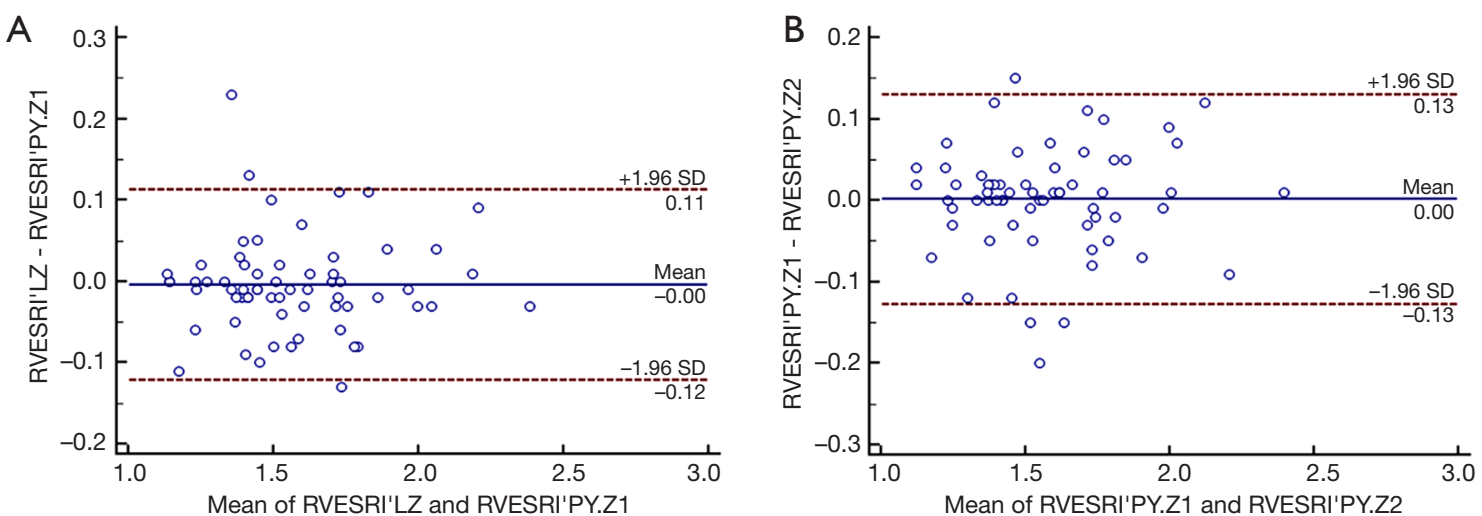

Figure 3 Reproducibility of right ventricular end-systolic remodeling index (RVESRI) measurements by Bland-Altman plots. (A) Measurements of RVESRI by 2 independent observers and (B) measurements of RVESRI twice by one observer.

\section{Discussion}

There are 3 main findings in the current study: (I) RVESRI measured by CMR has the advantage of simplicity and reproducibility without the need for the special post- processing software; (II) increased RVESRI closely correlates with other RV ventricular remodeling and dysfunctional metrics; (III) increased RVESRI correlates with elevated pulmonary arterial pressure and afterload, 
Table 3 Correlations of RV end-systolic remodeling index with other CMR functional metrics

\begin{tabular}{lcc}
\hline CMR functional metrics & Spearman correlation in total patients $(r)$ & Spearman correlation in patients with CTEPH $(r)$ \\
\hline RVEI & $-0.81(P<0.001)^{\star}$ & $-0.67(P<0.001)$ \\
RVEDVI $\left(\mathrm{mL} / \mathrm{m}^{2}\right)$ & $0.79(\mathrm{P}<0.001)^{\star}$ & $0.71(\mathrm{P}<0.001)^{\star}$ \\
RVESVI $\left(\mathrm{mL} / \mathrm{m}^{2}\right)$ & $0.83(\mathrm{P}<0.001)^{\star}$ & $0.62(\mathrm{P}<0.001)^{\star}$ \\
RVEF $(\%)$ & $-0.64(\mathrm{P}<0.001)^{\star}$ & $-0.59(\mathrm{P}<0.001)^{\star}$ \\
RVCO $(\mathrm{L} / \mathrm{min})$ & $-0.50(\mathrm{P}<0.001)^{\star}$ & $-0.42(\mathrm{P}=0.008)$ \\
RVMI $\left(\mathrm{g} / \mathrm{m}^{2}\right)$ & $0.67(\mathrm{P}<0.001)^{\star}$ & $0.37(\mathrm{P}=0.014)$ \\
RVGLS $(\%)$ & $0.62(\mathrm{P}<0.001)^{\star}$ & $0.41(\mathrm{P}=0.022)$ \\
RVGRS $(\%)$ & $-0.19(\mathrm{P}=0.118)$ & $-0.23(\mathrm{P}=0.137)$ \\
RVGCS $(\%)$ & $0.02(\mathrm{P}=0.998)$ & $0.18(\mathrm{P}=0.234)$ \\
LVEDVI $\left(\mathrm{mL} / \mathrm{m}^{2}\right)$ & $-0.39(\mathrm{P}=0.001)$ & $-0.22(\mathrm{P}=0.081)$ \\
LVESVI $\left(\mathrm{mL} / \mathrm{m}^{2}\right)$ & $-0.03(\mathrm{P}=0.851)$ & $-0.10(\mathrm{P}=0.265)$ \\
LVEF $(\%)$ & $-0.26(\mathrm{P}=0.037)$ & $-0.15(\mathrm{P}=0.118)$ \\
\hline
\end{tabular}

*, $\mathrm{P}<0.001$, Spearman correlation analysis of the RVESRI and other functional metrics; RVEI, right ventricular eccentricity index; RVESRI, RV end-systolic remodeling index; RVEDVI, RV end-diastolic volume/body surface area; RVESVI, RV end-systolic volume/body surface area; RVEF, RV ejection fraction; RVCO, RV cardiac output; RVMI, RV myocardial mass/body surface area; RVGRS, RV global radial strain; RVGCS, RV global circumferential strain; RVGLS, RV global longitudinal strain; LVEDVI, LV end-diastolic volume/body surface area; LVESVI, LV end-systolic volume/body surface area; LVEF, LV ejection fraction.
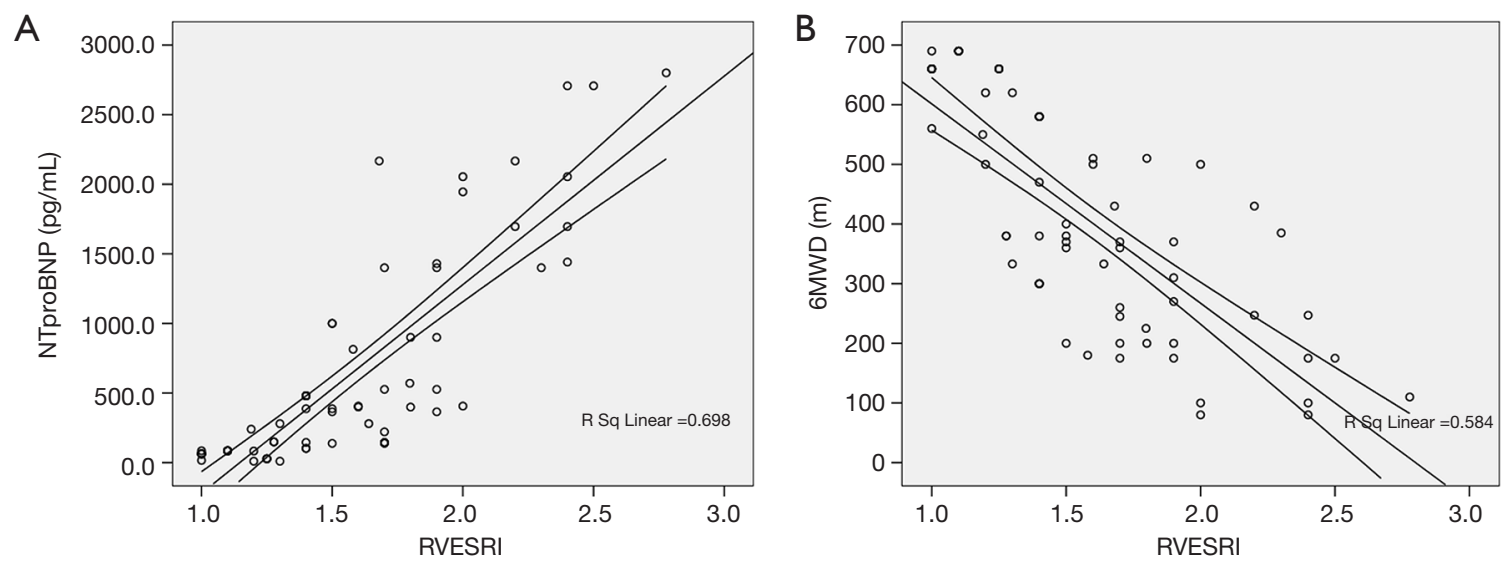

Figure 4 Correlation analysis of the right ventricular end-systolic remodeling index (RVESRI) with plasma N-terminal pro-brain natriuretic peptide (NT-proBNP) and 6-minute walk distance (6MWD). (A) RVESRI positively correlated with plasma NT-proBNP (r=0.85, $\mathrm{P}<0.001$ ). (B) RVESRI negatively correlated with $6 \mathrm{MWD}(\mathrm{r}=-0.79, \mathrm{P}<0.001)$.

while RVESRI $>1.35$ has a sensitivity of $97.83 \%$ and specificity of $83.33 \%$ in predicting MPAP $\geq 25 \mathrm{mmHg}$.

CTEPH is a potentially curable $\mathrm{PH}$ disease and can be treated by pulmonary endarterectomy or pulmonary balloon angioplasty. CMR studies have emphasized the predictive value of RVEF and RV end-diastolic and end- systolic volumes (10-12). The anatomical complexity of the right ventricle made the measurement of RVEF on CMR more time-consuming and complicated than the left ventricle. Amsallem et al. (5) proposed that RVESRI has the advantage of scaling using the same dimension and being less influenced by obesity. Since 4-chamber cine images 

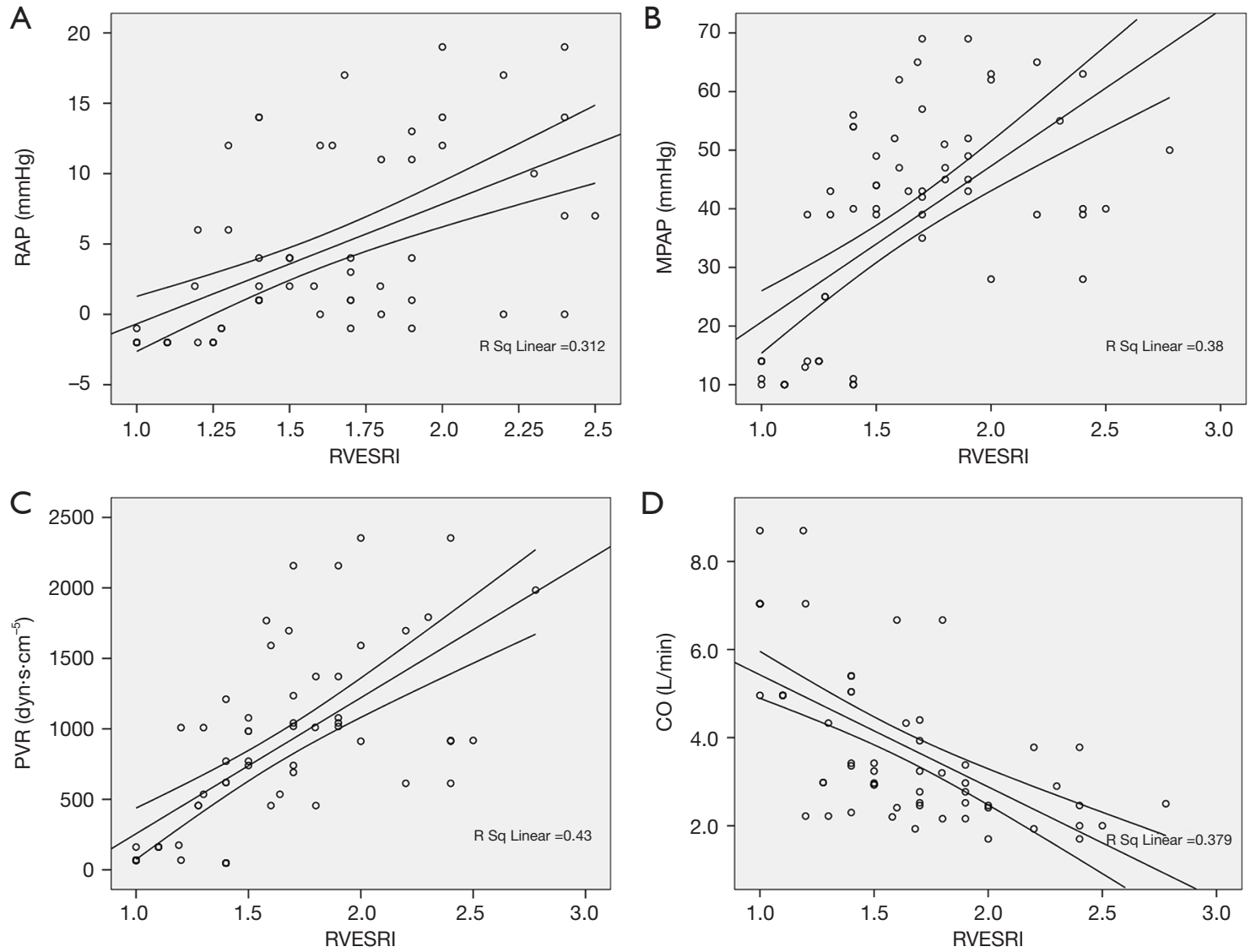

Figure 5 Correlation analysis of right ventricular end-systolic remodeling index (RVESRI) with hemodynamics. (A) RVESRI positively correlated with right atrial pressure (RAP) $(\mathrm{r}=0.64, \mathrm{P}<0.001)$. (B) RVESRI positively correlated with mean pulmonary arterial pressure (MPAP) $(r=0.65, \mathrm{P}<0.001)$. (C) RVESRI positively correlated with pulmonary vascular resistance (PVR) $(r=0.69, \mathrm{P}<0.001)$. (D) RVESRI negatively correlated with cardiac output $(\mathrm{CO})(\mathrm{r}=-0.64, \mathrm{P}<0.001)$.

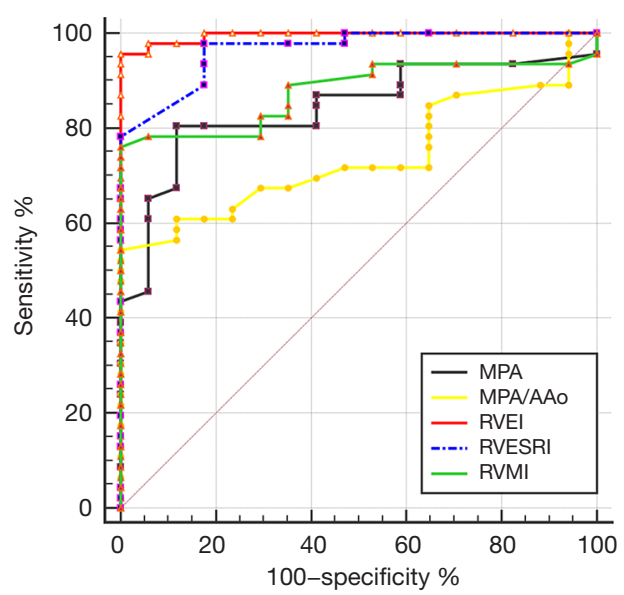

Figure 6 Performance of right ventricular end-systolic remodeling index (RVESRI), right ventricular eccentricity index (RVEI), right ventricular myocardial mass index (RVMI), the diameter of the main pulmonary artery (MPA), and the ratio of the MPA and ascending aorta diameter (MPA/AAo) for the prediction of mean pulmonary artery pressure $\geq 25 \mathrm{mmHg}$ as indicated by receiver operating characteristic curves. 
Table 4 AUCs of CMR metrics in the prediction of pulmonary hypertension (mean pulmonary arterial pressure $\geq 25 \mathrm{mmHg}$ )

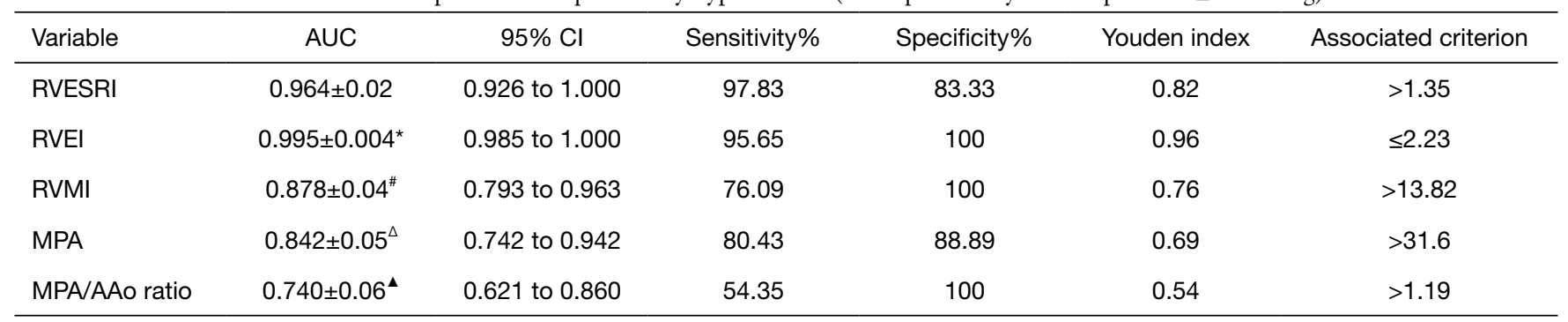

*, the comparison of RVESRI and RVEI, $Z=1.635, P=0.102$; \#, the comparison of RVESRI and RVMI, $Z=2.192, P=0.03 ; \Delta$, the comparison of RVESRI and MPA, Z=2.269, $P=0.02 ; \boldsymbol{\Lambda}$, the comparison of RVESRI and MPAAAo ratio, $Z=3.826, P<0.001$. AUC, area under the ROC curve; CMR, cardiac magnetic resonance; RVESRI, RV end-systolic remodeling index; RVEl, right ventricular eccentricity index; RVMI, RV myocardial mass index; MPA, the diameter of the main pulmonary artery; MPAAAo ratio, the ratio of the MPA and ascending aorta diameter.

on CMR can clearly demonstrate the temporal changes of the ventricular walls and chambers, our results indicated that the measurement of RVESRI on CMR is simple and reproducible.

More and more studies (13-15) have applied CMR in the evaluation of $\mathrm{PH}$ and have identified a range of valuable metrics, among which RVEF is an important prognostic factor for PH. In patients with CTEPH, RVESRI correlated with RVEDVI, RVESVI, and RVEF, indicating that RVESRI is the functional marker of RV systolic performance. In pediatric PAH, RVESRI is a useful marker in assessing right ventricular remodeling and dilation (16). With the elevation of right ventricular afterload, ventricular adaptive remodeling gradually progresses to maladaptive remodeling, which manifests as right ventricular wall hypertrophy and dilation. Progressive right ventricular remodeling in $\mathrm{PAH}$ is associated with a parallel decline in transversal and longitudinal shortening until a floor effect is reached for longitudinal shortening. Subsequently, the further reduction of right ventricular function is due to progressive leftward septal displacement and the increased RVESRI. Thus, ventricular strain can identify regional and global dysfunction. Some studies (17-19) showed that RVGLS was a remodeling marker and one of the strongest predictors of outcome in PH, and RVGLS, including right ventricular free wall and interventricular septum, showed a better correlation with RVEF by CMR. Recently, the utility of three-dimensional right ventricular strain was reported to be more accurate than two-dimensional strain in CTEPH (20). However, measurement depends on special post-processing software. In the current study, RVGLS was reduced in patients with CTEPH, and RVESRI was correlated with RVGLS and RVEF.
Moreover, CMR-derived RVEI (9) appears to have useful diagnostic and prognostic value in $\mathrm{PAH}$, and provides incremental value to the risk stratification strategy. There was a significant negative correlation between RVESRI and RVEI. Moreover, the AUCs of RVESRI and RVEI in predicting $\mathrm{mPAP} \geq 25 \mathrm{mmHg}$ were comparable. These findings indicate that RVESRI on CMR is a remodeling marker like RVEI, which is easy to measure, and could provide a prognostic assessment of patients with CTEPH.

In terms of circulating blood biomarkers, BNP and NTproBNP have been predictive of $\mathrm{PH}$ patients' outcomes $(21,22)$. Clinical studies of PAH have used the change in 6MWD as a clinical end point (23). RVESRI was positively associated with NT-proBNP and negatively associated with $6 \mathrm{MWD}$, which indicated that it could be a prognostic marker. RHC is considered the gold standard for the diagnosis of $\mathrm{PH}$ and the assessment of hemodynamic severity. However, frequent or serial invasive testing is not practical. RVESRI correlated with RAP, PAP, PVR, and CO measured with RHC. Dilatation of the MPA is a common characteristic of $\mathrm{PAH}$, and the identification of $\mathrm{PA}$ dilation on CT or MR of the chest performed to assess patients with nonspecific cardiorespiratory symptoms may raise the possibility of PH (24-27). Thus, we compared RVESRI, MPA, and MPA/AAo ratio in predicting $\mathrm{PH}$, and the ROC curve indicated that RVESRI $>1.35$ had a higher sensitivity, specificity, and AUC value in identifying $\mathrm{mPAP} \geq 25 \mathrm{mmHg}$ compared to MPA and MPA/AAo ratio. However, in total patients and CTEPH patients, RVESRI moderately $(\mathrm{r}=0.65)$ and weakly ( $\mathrm{r}=0.33$ ) correlated with MPAP respectively, indicating that even though RVESRI might be a good parameter for predicting increased MPAP, it does not necessarily have to be accurate in estimating the MPAP 
value. Based on the present observations, RVESRI can be used to identify CTEPH patients from CTE patients and assess RV afterload (e.g., pulmonary pressure, PVR), stress (e.g., strain, NT-proBNP), and functional adaptation (e.g., RVEF, RVESVI) for patients with CTEPH.

\section{Limitations}

It should be noted that there are several limitations in our current study. As a retrospective study, we included patients with confirmed CTEPH and CTE. However, most patients were excluded because they had no CMR or a longer time interval of CMR and RHC, and only a small number of patients at a single center were included. This led to the drastic difference in the hemodynamic parameters of the CTE vs. CTEPH groups which was the important limitation of this study. Therefore, future studies with larger numbers of patients are required to determine the actual utility of RVESRI for the evaluation of CTEPH. Predictors of perioperative mortality in patients with operable CTEPH are useful in planning treatment, and whether RVESRI is a prognostic parameter needs to be determined. Our study only included certain CTEPH patients, a subgroup of $\mathrm{PH}$, and whether these findings can be extrapolated to other groups of $\mathrm{PH}$ patients such as those with $\mathrm{PH}$ due to lung diseases and/or hypoxia or $\mathrm{PH}$ due to left heart disease need further research in the future.

\section{Conclusions}

In our study, RVESRI can be easily and reproducibly assessed on cine images of CMR, which are acquired in virtually all patients undergoing CMR. Moreover, CMRderived RVESRI may assist in evaluating CTEPH.

\section{Acknowledgments}

Funding: This work is supported by the National Natural Science Foundation of China (Grant No. 81871328), Beijing Nature Science Foundation (Grant No. 7182149), Youth Talents project of Chinese Academy of Medical Science (Grant No. 2018RC320013), and Beijing Science and Technology Commission Pharmaceutical and Technology Innovation Project (Grant No. Z181100001918034).

\section{Footnote}

Conflicts of Interest: All authors have completed the ICMJE uniform disclosure form (available at https://dx.doi. org/10.21037/qims-21-385). The authors have no conflicts of interest to declare.

Ethical Statement: The authors are accountable for all aspects of the work in ensuring that questions related to the accuracy or integrity of any part of the work are appropriately investigated and resolved. The study was approved by the ethics committee of China-Japan Friendship Hospital (IRB No.2017-24), and was performed following the latest version of the Declaration of Helsinki (as revised in 2013) for Medical Research involving human subjects. Informed consent was obtained from all patients.

Open Access Statement: This is an Open Access article distributed in accordance with the Creative Commons Attribution-NonCommercial-NoDerivs 4.0 International License (CC BY-NC-ND 4.0), which permits the noncommercial replication and distribution of the article with the strict proviso that no changes or edits are made and the original work is properly cited (including links to both the formal publication through the relevant DOI and the license). See: https://creativecommons.org/licenses/by-nc-nd/4.0/.

\section{References}

1. Galiè N, Humbert M, Vachiery JL, Gibbs S, Lang I, Torbicki A, et al. 2015 ESC/ERS Guidelines for the diagnosis and treatment of pulmonary hypertension: The Joint Task Force for the Diagnosis and Treatment of Pulmonary Hypertension of the European Society of Cardiology (ESC) and the European Respiratory Society (ERS): Endorsed by: Association for European Paediatric and Congenital Cardiology (AEPC), International Society for Heart and Lung Transplantation (ISHLT). Eur Heart J 2016;37:67-119.

2. Shemin RJ. Surgical Embolectomy for Massive and Submassive Pulmonary Embolism and Pulmonary Thromboendarterectomy for Chronic Thromboembolic Pulmonary Hypertension. Tech Vasc Interv Radiol 2017;20:175-8.

3. Karyofyllis P, Demerouti E, Papadopoulou V, Voudris V, Matsubara H. Balloon Pulmonary Angioplasty as a Treatment in Chronic Thromboembolic Pulmonary Hypertension: Past, Present, and Future. Curr Treat Options Cardiovasc Med 2020;22:7.

4. Baillie TJ, Sidharta S, Steele PM, Worthley SG, Willoughby S, Teo K, Sanders P, Nicholls SJ, Worthley 
MI. The predictive capabilities of a novel cardiovascular magnetic resonance derived marker of cardiopulmonary reserve on established prognostic surrogate markers in patients with pulmonary vascular disease: results of a longitudinal pilot study. J Cardiovasc Magn Reson 2017;19:3.

5. Amsallem M, Sweatt AJ, Aymami MC, Kuznetsova T, Selej M, Lu H, Mercier O, Fadel E, Schnittger I, McConnell MV, Rabinovitch M, Zamanian RT, Haddad F. Right Heart End-Systolic Remodeling Index Strongly Predicts Outcomes in Pulmonary Arterial Hypertension: Comparison With Validated Models. Circ Cardiovasc Imaging 2017;10:e005771.

6. Swift AJ, Capener D, Johns C, Hamilton N, Rothman A, Elliot C, Condliffe R, Charalampopoulos A, Rajaram S, Lawrie A, Campbell MJ, Wild JM, Kiely DG. Magnetic Resonance Imaging in the Prognostic Evaluation of Patients with Pulmonary Arterial Hypertension. Am J Respir Crit Care Med 2017;196:228-39.

7. Baggen VJ, Leiner T, Post MC, van Dijk AP, RoosHesselink JW, Boersma E, Habets J, Sieswerda GT. Cardiac magnetic resonance findings predicting mortality in patients with pulmonary arterial hypertension: a systematic review and meta-analysis. Eur Radiol 2016;26:3771-80.

8. Wang L, Liu M, Zhang PY, Dai JZ, Ma HY, Tao XC, Xie WM, Wan J, Jing A. Analysis of right ventricular flow with 4-dimensional flow cardiovascular magnetic resonance imaging in patients with pulmonary arterial hypertension. Quant Imaging Med Surg 2021;11:3655-65.

9. Wang L, Chen X, Wan K, Gong C, Li W, Xu Y, Wang J, He J, Wen B, Han Y, Zeng R, Chen Y. Diagnostic and prognostic value of right ventricular eccentricity index in pulmonary artery hypertension. Pulm Circ 2020;10:2045894019899778.

10. Lewis RA, Johns CS, Cogliano M, Capener D, Tubman E, Elliot CA, et al. Identification of Cardiac Magnetic Resonance Imaging Thresholds for Risk Stratification in Pulmonary Arterial Hypertension. Am J Respir Crit Care Med 2020;201:458-68.

11. Aryal SR, Sharifov OF, Lloyd SG. Emerging role of cardiovascular magnetic resonance imaging in the management of pulmonary hypertension. Eur Respir Rev 2020;29:190138.

12. Alabed S, Shahin Y, Garg P, Alandejani F, Johns CS, Lewis RA, Condliffe R, Wild JM, Kiely DG, Swift AJ. Cardiac-MRI Predicts Clinical Worsening and Mortality in Pulmonary Arterial Hypertension: A Systematic
Review and Meta-Analysis. JACC Cardiovasc Imaging 2021;14:931-42.

13. van Wolferen SA, Marcus JT, Boonstra A, Marques KM, Bronzwaer JG, Spreeuwenberg MD, Postmus PE, VonkNoordegraaf A. Prognostic value of right ventricular mass, volume, and function in idiopathic pulmonary arterial hypertension. Eur Heart J 2007;28:1250-7.

14. van de Veerdonk MC, Kind T, Marcus JT, Mauritz GJ, Heymans MW, Bogaard HJ, Boonstra A, Marques KM, Westerhof N, Vonk-Noordegraaf A. Progressive right ventricular dysfunction in patients with pulmonary arterial hypertension responding to therapy. J Am Coll Cardiol 2011;58:2511-9.

15. Yamada Y, Okuda S, Kataoka M, Tanimoto A, Tamura Y, Abe T, Okamura T, Fukuda K, Satoh T, Kuribayashi S. Prognostic value of cardiac magnetic resonance imaging for idiopathic pulmonary arterial hypertension before initiating intravenous prostacyclin therapy. Circ J 2012;76:1737-43.

16. Koestenberger M, Avian A, Chouvarine P, Gamillscheg A, Cvirn G, Schweintzger S, Kurath-Koller S, Cantinotti M, Hohmann D, Hansmann G. Right ventricular endsystolic remodeling index in the assessment of pediatric pulmonary arterial hypertension. The European Pediatric Pulmonary Vascular Disease Network (EPPVDN). Pediatr Res 2020;88:285-92.

17. de Siqueira ME, Pozo E, Fernandes VR, Sengupta PP, Modesto K, Gupta SS, Barbeito-Caamaño C, Narula J, Fuster V, Caixeta A, Sanz J. Characterization and clinical significance of right ventricular mechanics in pulmonary hypertension evaluated with cardiovascular magnetic resonance feature tracking. J Cardiovasc Magn Reson 2016;18:39.

18. Fine NM, Chen L, Bastiansen PM, Frantz RP, Pellikka PA, Oh JK, Kane GC. Outcome prediction by quantitative right ventricular function assessment in 575 subjects evaluated for pulmonary hypertension. Circ Cardiovasc Imaging 2013;6:711-21.

19. Park JH, Park MM, Farha S, Sharp J, Lundgrin E, Comhair S, Tang WH, Erzurum SC, Thomas JD. Impaired Global Right Ventricular Longitudinal Strain Predicts Long-Term Adverse Outcomes in Patients with Pulmonary Arterial Hypertension. J Cardiovasc Ultrasound 2015;23:91-9.

20. Kawakubo M, Yamasaki Y, Kamitani T, Sagiyama K, Matsuura Y, Hino T, Abe K, Hosokawa K, Yabuuchi H, Honda H. Clinical usefulness of right ventricular 3D area strain in the assessment of treatment effects of balloon 
pulmonary angioplasty in chronic thromboembolic pulmonary hypertension: comparison with 2D featuretracking MRI. Eur Radiol 2019;29:4583-92.

21. Fijalkowska A, Kurzyna M, Torbicki A, Szewczyk G, Florczyk M, Pruszczyk P, Szturmowicz M. Serum $\mathrm{N}$-terminal brain natriuretic peptide as a prognostic parameter in patients with pulmonary hypertension. Chest 2006;129:1313-21.

22. Reesink HJ, Tulevski II, Marcus JT, Boomsma F, Kloek JJ, Vonk Noordegraaf A, Bresser P. Brain natriuretic peptide as noninvasive marker of the severity of right ventricular dysfunction in chronic thromboembolic pulmonary hypertension. Ann Thorac Surg 2007;84:537-43.

23. Farber HW, Miller DP, McGoon MD, Frost AE, Benton WW, Benza RL. Predicting outcomes in pulmonary

Cite this article as: Zhang L, Dai J, Zhang P, Ma H, Tao X, Zhen Y, Liu X, Xie W, Wan J, Liu M. Right ventricular endsystolic remodeling index on cardiac magnetic resonance imaging: comparison with other functional markers in patients with chronic thromboembolic pulmonary hypertension. Quant Imaging Med Surg 2022;12(2):894-905. doi: 10.21037/qims-21385 arterial hypertension based on the 6-minute walk distance. J Heart Lung Transplant 2015;34:362-8.

24. Boerrigter B, Mauritz GJ, Marcus JT, Helderman F, Postmus PE, Westerhof N, Vonk-Noordegraaf A. Progressive dilatation of the main pulmonary artery is a characteristic of pulmonary arterial hypertension and is not related to changes in pressure. Chest 2010;138:1395-401.

25. Peña E, Dennie C, Veinot J, Muñiz SH. Pulmonary hypertension: how the radiologist can help. Radiographics 2012;32:9-32.

26. Frazier AA, Burke AP. The imaging of pulmonary hypertension. Semin Ultrasound CT MR 2012;33:535-51.

27. Raymond TE, Khabbaza JE, Yadav R, Tonelli AR. Significance of main pulmonary artery dilation on imaging studies. Ann Am Thorac Soc 2014;11:1623-32. 